\title{
Value Distribution and Uniqueness Results of Zero-Order Meromorphic Functions to Their q-Shift
}

\author{
Haiwa Guan, ${ }^{1}$ Gang Wang, ${ }^{2}$ and Qiuqin Luo ${ }^{3}$ \\ ${ }^{1}$ Department of Public Teaching, Wenzhou Vocational College of Science and Technology, Zhejiang, \\ Wenzhou 325000, China \\ ${ }^{2}$ Department of Science and Humanities, Shandong Transport Vocational College, Shandong, \\ Weifang 261206, China \\ ${ }^{3}$ Department of Educational Administration and Supervision, Wenzhou Vocational College of \\ Science and Technology, Zhejiang, Wenzhou 325000, China
}

Correspondence should be addressed to Gang Wang, wangganggangwang@126.com

Received 21 June 2012; Accepted 18 September 2012

Academic Editor: Risto Korhonen

Copyright (C) 2012 Haiwa Guan et al. This is an open access article distributed under the Creative Commons Attribution License, which permits unrestricted use, distribution, and reproduction in any medium, provided the original work is properly cited.

We investigate value distribution and uniqueness problems of meromorphic functions with their $q$-shift. We obtain that if $f$ is a transcendental meromorphic (or entire) function of zero order, and $Q(z)$ is a polynomial, then $a f^{n}(q z)+f(z)-Q(z)$ has infinitely many zeros, where $q \in \mathbb{C} \backslash\{0\}, a$ is nonzero constant, and $n \geq 5$ (or $n \geq 3$ ). We also obtain that zero-order meromorphic function share is three distinct values IM with its $q$-difference polynomial $P(f)$, and if $\limsup _{r \rightarrow \infty}(N(r, f) / T(r, f))<1$, then $f \equiv P(f)$.

\section{Introduction and Main Results}

A function $f(z)$ is called meromorphic function if it is analytic in the complex plane except at isolated poles. It is assumed that the reader is familiar with the standard symbols and fundamental results of Nevanlinna theory such as the characteristic function $T(r, f)$, proximity function $m(r, f)$, and the counting function $N(r, f)$, see [1-3]. Let us recall the definition of the order and the zeros exponent convergence of function $f$. The order of meromorphic function $f$ is said by

$$
\rho(f)=\limsup _{r \rightarrow \infty} \frac{\log T(r, f)}{\log r}
$$


The zeros of exponent convergence of meromorphic function $f$ is said by

$$
\lambda(f)=\limsup _{r \rightarrow \infty} \frac{\log N(r, f)}{\log r} .
$$

In 1959, Hayman proved the following Theorems.

Theorem A (see [4], Theorem 8). Let $f$ be a transcendental entire function, and let $n \geq 3$ be an integer and a be a nonzero constant. Then $f^{\prime}(z)-a f(z)$ assumes all finite values infinitely often.

Theorem B (see [4], Theorem 9). Let $f$ be a transcendental meromorphic function, and let $n \geq 5$ be an integer and a be a nonzero constant. Then $f^{\prime}(z)-a f(z)$ assumes all finite values infinitely often.

Recently the difference variant of the Nevanlinna theory has been established independently in $[5,6]$. Using these theories, value distribution theory uniqueness theory of difference polynomials of finite order transcendental meromorphic functions has been studied as well. We recall the following result by Liu and Laine.

Theorem C (see [7], Theorem 1.1). Let $f$ be a transcendental entire function of finite order not of period $c$, where $c$ is a nonzero constant, and let $s(z)$ be a nonzero small function of $f$. Then the difference polynomial $f^{n}(z)+f(z+c)-f(z)-s(z)$ has infinitely many zeros in the complex plane provided that $n \geq 3$.

In 2010, Chen considered the difference counterpart of Hayman's theorem and porved an almost direct difference analogue of Hayman's theorem.

Theorem D (see [8], Theorem 1.1). Let $f$ be a transcendental entire function of finite order not of period $c$, and let $a(\neq 0), b, c(\neq 0)$ be three complex numbers. Then $\Psi_{n}(z)=f(z+c)-f(z)-a f^{n}(z)$ assumes all finite values infinitely often, provided that $n \geq 3$ and for every $b$ one has $\lambda\left(\Psi_{n}(z)-b\right)=$ $\rho(f)$.

In this paper, we consider the value distribution of zero-order meromorphic functions with their $q$-shirt and prove the following results.

Theorem 1.1. Let $f$ be a transcendental meromorphic function of zero order, and let $Q(z)$ be a polynomial. If $n$ is an integer and $n \geq 4$, then $a f^{n}(q z)+f(z)-Q(z)$ has infinitely many zeros, where $q \in \mathbb{C} \backslash\{0\}$ and $a$ is nonzero constant.

Theorem 1.2. Let $f$ be a transcendental entire function of zero order, and let $Q(z)$ be a polynomial. If $n$ is an integer and $n \geq 3$, then $a f^{n}(q z)+f(z)-Q(z)$ has infinitely many zeros, where $q \in \mathbb{C} \backslash\{0\}$, and $a$ is nonzero constant.

It is well known that two meromorphic functions must be equal, if they share five distinct values. Recently, Heittokangas et al. research the uniqueness of meromorphic functions with their shifts in [6]. They got that if $f(z)$ and $f(z+c)$ share three distinct values, where $f(z)$ is finite order, then $f(z)=f(z+c)$. In this paper, we want to get some results on uniqueness of $f(z)$ and $f(q z)$, where $f(z)$ is zero order and $q \in \mathbb{C} \backslash\{0,1\}$. Let us recall the notation of $q$-difference which is written by $\nabla_{q}=f(q z)-f(z)$. 
Theorem 1.3. Let $f$ be a meromorphic function of zero order, let $q \in \mathbb{C} \backslash\{0,1\}$, and let $a_{1}, a_{2}, a_{3} \in \mathbb{C}$ be three distinct values.

(a) If $f(z)$ and $f(q z)$ share $a_{1}, a_{2}, a_{3} C M$, then $f(z)=f(q z)$ for all $z \in \mathbb{C}$.

(b) If $f(z)$ and $f(q z)$ share $a_{1}, a_{2} C M$, and if

$$
\operatorname{Limsup}_{r \rightarrow \infty} \frac{N(r, f)}{T(r, f)}<1,
$$

then $f(z)=f(q z)$ for all $z \in \mathbb{C}$.

Corollary 1.4. Let $f$ be an entire function of zero order, let $q \in \mathbb{C} \backslash\{0,1\}$, and let $a_{1}, a_{2} \in \mathbb{C}$ be two distinct values.

(a) If $f(z)$ and $f(q z)$ share $a_{1}, a_{2} C M$, then $f(z)=f(q z)$ for all $z \in \mathbb{C}$.

(b) If $f(z)$ and $f(q z)$ share $a_{1} C M$, and if

$$
\limsup _{r \rightarrow \infty} \frac{N(r, f)}{T(r, f)}<1
$$

then $f(z)=f(q z)$ for all $z \in \mathbb{C}$.

Corollary 1.5. Let $f$ be a meromorphic function of zero order, and let $q \in \mathbb{C} \backslash\{0,1\}$. If $f(z)$ and $f(q z)$ share $\infty C M$ and a constant $a \in \mathbb{C} C M$, and if there exists a constant $b \in \mathbb{C} \backslash\{a\}$ such that

$$
\limsup _{r \rightarrow \infty} \frac{N(r, 1 /(f-b))}{T(r, f)}<1
$$

then $f(z)=f(q z)$ for all $z \in \mathbb{C}$.

Theorem 1.6. Let $f$ be a meromorphic function of zero order, let $q \in \mathbb{C} \backslash\{0,1\}$, and let

$$
P(f)=b_{k}(z) f\left(q^{k} z\right)+\cdots+b_{1}(z) f(q z)+b_{0}(z) f(z)
$$

where $b_{k}, \ldots, b_{0}$ are constants. Let $n \in\{1, \ldots, k+1\}$ be the number of nonzero coefficients of the $q$-difference polynomial $P(f)-f$. If $f$ and $P(f)$ share three distinct finite values $a_{1}, a_{2}, a_{3} I M$, and if

$$
\limsup _{r \rightarrow \infty} \frac{N(r, f)}{T(r, f)}<1
$$

then $f \equiv P(f)$. 
Corollary 1.7. Let $f$ be a meromorphic function of zero order, let $q \in \mathbb{C} \backslash\{0,1\}$, and let $a_{1}, a_{2}, a_{3} \in \mathbb{C}$ be three distinct finite values.

(a) If $f(z)$ and $f(q z)$ share $a_{1}, a_{2}, a_{3}$ IM, and if

$$
\limsup _{r \rightarrow \infty} \frac{N(r, f)}{T(r, f)}<\frac{1}{2}
$$

then $f(z)=f(q z)$ for all $z \in \mathbb{C}$.

(b) If $f(z)$ and $f(q z)$ share $\infty I M$ and two constants $a_{1}, a_{2} \in \mathbb{C} I M$, and if there exists $a$ constant $b \in \mathbb{C} \backslash\left\{a_{1}, a_{2}\right\}$ such that

$$
\limsup _{r \rightarrow \infty} \frac{N(r, 1 /(f-b))}{T(r, f)}<\frac{1}{2}
$$

then $f(z)=f(q z)$ for all $z \in \mathbb{C}$.

Corollary 1.8. Let $f$ be an entire function of zero order, let $q \in \mathbb{C} \backslash\{0,1\}$, and let $a_{1}, a_{2}, a_{3} \in \mathbb{C}$ be three distinct finite values.

(a) If $f(z)$ and $f(q z)$ share $a_{1}, a_{2}, a_{3} I M$, then $f(z)=f(q z)$ for all $z \in \mathbb{C}$.

(b) If $f(z)$ and $f(q z)$ share $a_{1}, a_{2} \in \mathbb{C} I M$, and if there exists a constant $b \in \mathbb{C} \backslash\left\{a_{1}, a_{2}\right\}$ such that

$$
\limsup _{r \rightarrow \infty} \frac{N(r, 1 /(f-b))}{T(r, f)}<\frac{1}{2}
$$

then $f(z)=f(q z)$ for all $z \in \mathbb{C}$.

\section{Auxiliary Results}

The following auxiliary results will be instrumental in proving the theorems.

Lemma 2.1 (see [9], Theorem 1.2). Let $f(z)$ be a nonconstant zero-order meromorphic function, and $q \in \mathbb{C} \backslash\{0\}$. Then

$$
m\left(r, \frac{f(q z)}{f(z)}\right)=S_{q}(r, f) .
$$

Lemma 2.2 (see [9], Theorem 3.1). Let $f$ be a non-constant meromorphic functions of zero order, let $q \in \mathbb{C} \backslash\{0,1\}$, and let $a_{1}, \ldots, a_{p} \in \mathbb{C}$, where $p \geq 2$, be distinct points. Then

$$
m(r, f)+\sum_{k=1}^{p} m\left(r, \frac{1}{f-a_{k}}\right) \leq 2 T(r, f)-N_{\text {pair }}(r, f)+S_{q}(r, f)
$$


where

$$
N_{\text {pair }}(r, f):=2 N(r, f)-N\left(r, \nabla_{q} f\right)+N\left(r, \frac{1}{\nabla_{q} f}\right) .
$$

Lemma 2.3 (see [10], Theorem 1.1). Let $f(z)$ be a non-constant zero-order meromorphic function, and $q \in \mathbb{C} \backslash\{0\}$. Then

$$
T(r, f(q z))=T(r, f)+S_{q}(r, f) .
$$

Lemma 2.4 (see [10], Theorem 1.3). Let $f(z)$ be a non-constant zero-order meromorphic function, and $q \in \mathbb{C} \backslash\{0\}$. Then

$$
N(r, f(q z))=N(r, f)+S_{q}(r, f)
$$

Lemma 2.5 (see [11], Lemma 4). If $T: \mathbb{R}^{+} \rightarrow \mathbb{R}^{+}$is a piecewise continuous increasing function such that

$$
\lim _{r \rightarrow \infty} \frac{\log T(r)}{\log r}=0
$$

then the set

$$
E:=\left\{r: T\left(C_{1} r\right) \geq C_{2} T(r)\right\}
$$

has logarithmic density 0 for all $C_{1}>1$ and $C_{2}>1$.

\section{Proof of Theorem 1.1}

Let us put

$$
\Psi(z):=a f^{n}(q z)+f(z)-Q(z)
$$

Hence, $\Psi(z)$ is not a constant identity. If not, let us suppose that $\Psi(z) \equiv c$, where $c$ is a constant, and then

$$
a f^{n}(q z) \equiv Q(z)+c-f(z),
$$

which give us

$$
n T(r, f(q z))=T(r, f)+O(\log r) .
$$


By using Lemma 2.3, we have $n \leq 1$, which contradicts the assumption $n \geq 5$. Hence $\Psi(z) \not \equiv c$. By taking logarithmic derivative on two sides of (3.1), we have

$$
\frac{\Psi^{\prime}(z)}{\Psi(z)}=\frac{\left(a f^{n}(q z)+f(z)-Q(z)\right)^{\prime}}{a f^{n}(q z)+f(z)-Q(z)} .
$$

If

$$
\frac{\left(f^{n}(q z)\right)^{\prime}}{f^{n}(q z)}-\frac{\Psi^{\prime}(z)}{\Psi(z)} \equiv 0
$$

then by integrating two sides of which, we have $\Psi(z)=b f^{n}(q z)$, where $b$ is a nonzero constant, and hence

$$
(b-a) f^{n}(q z)=f(z)-Q(z) .
$$

If $b=a$, then $f(z)=Q(z)$, which is contradiction with $f(z)$, is transcendental function. If $b \neq a$, then Lemma 2.3 implies that $n=1$, which is impossible. Therefore, we can write (3.4) as

$$
a f^{n}(q z)=\frac{\left(\Psi^{\prime}(z) / \Psi(z)\right)(f(z)-Q(z))-(f(z)-Q(z))^{\prime}}{\left(f^{n}(q z)\right)^{\prime} / f^{n}(q z)-\Psi^{\prime}(z) / \Psi(z)}
$$

Let us put

$$
\Psi_{1}(z):=\frac{\Psi^{\prime}(z)}{\Psi(z)}(f(z)-Q(z))-(f(z)-Q(z))^{\prime}
$$

Now we consider the poles of $\Psi_{1}(z)$. The poles of $\Psi_{1}(z)$ come from the zeros of $\Psi(z)$ and the poles of $f(q z), f(z)$, and $Q(z)$. If $z_{0}$ is a zero of $\Psi(z)$ or a pole of $f(q z)$, but not a pole of $f(z)$, then $z_{0}$ is a simple pole of $\Psi(z)$. If $z_{0}$ is a common pole of $f(q z)$ and $f(z)$ with multiplicities of $k$ and $l$, respectively, then $z_{0}$ is a pole of $\Psi_{1}(z)$ with multiplicity no more than $l+1$. If $z_{0}$ is a pole of $f(z)$ but not a pole of $f(q z)$, we obtain that $z_{0}$ is at most a simple pole of $\Psi_{1}(z)$ by using (3.7). Hence, Lemma 2.5 implies that

$$
\begin{aligned}
N\left(r, \Psi_{1}\right) & \leq \bar{N}\left(r, \frac{1}{\Psi}\right)+\bar{N}(r, f(q z))+N(r, f(z))+O(\log r) \\
& \leq \bar{N}\left(r, \frac{1}{\Psi}\right)+\bar{N}(r, f)+N(r, f(z))+S_{q}(r, f) .
\end{aligned}
$$

Let us put

$$
\Psi_{2}(z):=\frac{\left(f^{n}(q z)\right)^{\prime}}{f^{n}(q z)}-\frac{\Psi^{\prime}(z)}{\Psi(z)}
$$


Now, let us consider the pole of $\Psi_{2}(z)$. The poles of $\Psi_{2}(z)$ come from the poles of $f(q z)$ and $f(z)$ and the zeros of $f(q z)$ and $\Psi(z)$. If $z_{0}$ is a zero of $\Psi(z)$, zero of $f(q z)$, or pole of $f(z)$, then $z_{0}$ is a simple pole of $\Psi_{2}(z)$. If $z_{0}$ is a pole of $f(q z)$ but not a pole of $f(z)$, by using Laurent series, we obtain that $\Psi_{2}(z)$ is analytic at $z_{0}$. Therefore, we have

$$
\begin{aligned}
N\left(r, \Psi_{2}\right) & \leq \bar{N}\left(r, \frac{1}{\Psi}\right)+\bar{N}\left(r, \frac{1}{f(q z)}\right)+\bar{N}(r, f(z))+O(\log r) \\
& \leq \bar{N}\left(r, \frac{1}{\Psi}\right)+\bar{N}\left(r, \frac{1}{f(z)}\right)+\bar{N}(r, f(z))+S_{q}(r, f)
\end{aligned}
$$

according to Lemma 2.5. In the coming (3.7) and Lemma 2.1, it implies that

$$
\begin{aligned}
n T(r, f)= & T\left(r, a f^{n}(q z)\right)+S_{q}(r, f)=T\left(r, \frac{\Psi_{1}}{\Psi_{2}}\right)+S_{q}(r, f) \\
\leq & m\left(r, \Psi_{1}\right)+m\left(r, \Psi_{2}\right)+N\left(r, \Psi_{1}\right)+N\left(r, \Psi_{2}\right)+S_{q}(r, f) \\
\leq & m(r, f)+m\left(r, \frac{\Psi^{\prime}}{\Psi}-\frac{(f-Q)^{\prime}}{f-Q}\right)+m\left(r, \Psi_{2}\right)+N\left(r, \Psi_{1}\right) \\
& +N\left(r, \Psi_{2}\right)+S_{q}(r, f) \\
\leq & 2 \bar{N}\left(r, \frac{1}{\Psi}\right)+\bar{N}\left(r, \frac{1}{f}\right)+\bar{N}(r, f(z))+2 N(r, f) \\
& +m(r, f)+S_{q}(r, f) .
\end{aligned}
$$

Therefore, we have

$$
(n-3) T(r, f) \leq 2 \bar{N}\left(r, \frac{1}{\Psi}\right)+S_{q}(r, f),
$$

which shows that $a f^{n}(q z)+f(z)-Q(z)$ has infinite zeros by $n \geq 4$.

\section{Proof of Theorem 1.2}

In the same manner as in the proof of Theorem 1.1, we have (3.1)-(3.7), noting that

$$
\Psi_{1}(z):=\frac{\Psi^{\prime}(z)}{\Psi(z)}(f(z)-Q(z))-(f(z)-Q(z))^{\prime} .
$$

Now consider the poles of $\Psi_{1}(z)$. The poles of $\Psi_{1}(z)$ come from the zeros of $\Psi(z)$ and the poles of $Q(z)$. If $z_{0}$ is a zero of $\Psi(z)$, then $z_{0}$ is a simple pole of $\Psi(z)$. Hence, Lemma 2.4 implies that

$$
N\left(r, \Psi_{1}\right) \leq \bar{N}\left(r, \frac{1}{\Psi}\right)+O(\log r) \leq \bar{N}\left(r, \frac{1}{\Psi}\right)+S_{q}(r, f)
$$


Let us put

$$
\Psi_{2}(z):=\frac{\left(f^{n}(q z)\right)^{\prime}}{f^{n}(q z)}-\frac{\Psi^{\prime}(z)}{\Psi(z)}
$$

Now, let us consider the pole of $\Psi_{2}(z)$. The poles of $\Psi_{2}(z)$ come from the poles of $Q(z)$ and the zeros of $f(q z)$ and $\Psi(z)$. If $z_{0}$ is a zero of $\Psi(z)$, zero of $f(q z)$, then $z_{0}$ is a simple pole of $\Psi_{2}(z)$. Therefore, we have

$$
\begin{aligned}
N\left(r, \Psi_{2}\right) & \leq \bar{N}\left(r, \frac{1}{\Psi}\right)+\bar{N}\left(r, \frac{1}{f(q z)}\right)+O(\log r) \\
& \leq \bar{N}\left(r, \frac{1}{\Psi}\right)+\bar{N}\left(r, \frac{1}{f(z)}\right)+S_{q}(r, f),
\end{aligned}
$$

according to Lemma 2.5. In the coming (3.7) and Lemma 2.1, it implies that

$$
\begin{aligned}
n T(r, f)= & T\left(r, a f^{n}(q z)\right)+S_{q}(r, f)=T\left(r, \frac{\Psi_{1}}{\Psi_{2}}\right)+S_{q}(r, f) \\
\leq & m\left(r, \Psi_{1}\right)+m\left(r, \Psi_{2}\right)+N\left(r, \Psi_{1}\right)+N\left(r, \Psi_{2}\right)+S_{q}(r, f) \\
\leq & m(r, f)+m\left(r, \frac{\Psi^{\prime}}{\Psi}-\frac{(f-Q)^{\prime}}{f-Q}\right)+m\left(r, \Psi_{2}\right)+N\left(r, \Psi_{1}\right) \\
& +N\left(r, \Psi_{2}\right)+S_{q}(r, f) \\
\leq & 2 \bar{N}\left(r, \frac{1}{\Psi}\right)+\bar{N}\left(r, \frac{1}{f}\right)+m(r, f)+S_{q}(r, f) .
\end{aligned}
$$

Therefore, we have

$$
(n-2) T(r, f) \leq 2 \bar{N}\left(r, \frac{1}{\Psi}\right)+S_{q}(r, f),
$$

which shows that $a f^{n}(q z)+f(z)-Q(z)$ has infinite zeros by $n \geq 3$.

\section{Proof of Theorem 1.3}

(a) Suppose first that $a_{1}, a_{2}, a_{3}$ are three distinct values, and assume conversely to the assertion that $\nabla_{q} \not \equiv 0$. Then Lemma 2.2 yields

$$
\sum_{k=1}^{3} m\left(r, \frac{1}{f-a_{k}}\right) \leq 2 T(r, f)+N\left(r, \nabla_{q} f\right)-2 N(r, f)-N\left(r, \frac{1}{\nabla_{q} f}\right)+S_{q}(r, f)
$$


and so

$$
T(r, f) \leq \sum_{k=1}^{3} N\left(r, \frac{1}{f-a_{k}}\right)+N(r, f(q z))-N(r, f)-N\left(r, \frac{1}{\nabla_{q} f}\right)
$$

Since $f(z)$ and $f(q z)$ share $a_{1}, a_{2}$, and $a_{3} \mathrm{CM}$, it follows that

$$
\sum_{k=1}^{3} N\left(r, \frac{1}{f-a_{k}}\right) \leq N\left(r, \frac{1}{\nabla_{q} f}\right)
$$

In addition, since $f$ is zero-order meromorphic function, from Lemma 2.4, Lemma 2.5 and equations (5.2) and (5.3), we have

$$
T(r, f)=S_{q}(r, f)
$$

which is impossible. This contradiction is only avoided when $\nabla_{q} f \equiv 0$.

Suppose that $a_{3}=\infty$ while $a_{1}$ and $a_{2}$ are distinct finite values. Similarly as above, $f(z)=f(q z)$ can be obtained. Therefore, $f(z)=f(q z)$ for all $z \in \mathbb{C}$.

(b) Assume that $\nabla_{q} f \not \equiv 0$. Similarly as above, Lemma 2.2 yields

$$
\begin{aligned}
m(r, f)+\sum_{k=1}^{2} m\left(r, \frac{1}{f-a_{k}}\right) \leq & 2 T(r, f)+N\left(r, \nabla_{q} f\right) \\
& -2 N(r, f)-N\left(r, \frac{1}{\nabla_{q} f}\right)+S_{q}(r, f)
\end{aligned}
$$

and therefore $m(r, f)=S_{q}(r, f)$. This together with the condition results in a contradiction. Hence $\nabla_{q} f \not \equiv 0$.

\section{Proof of Theorem 1.6}

Assume on the contrary to the assertion that $f \not \equiv P(f)$. In what follows, $\varepsilon>0$ is small enough and $R>0$ is large enough. From the condition, we have

$$
N(r, f) \leq \frac{1-2 \varepsilon}{n} T(r, f), \quad r \geq R,
$$


and this together with Lemma 2.4 and Lemma 2.1 gives

$$
\begin{aligned}
N\left(r, \frac{1}{P(f)-f}\right) & \leq T(r, P(f)-f)+O(1) \\
& \leq m(r, P(f)-f)+N(r, P(f)-f)+O(1) \\
& =m\left(r, f\left(\frac{P(f)}{f}-1\right)\right)+n N(r, f)+S_{q}(r, f) \\
& \leq T(r, f)+(n-1) N(r, f)+S_{q}(r, f), \quad r \geq R .
\end{aligned}
$$

Therefore, by the sharing assumption,

$$
\begin{aligned}
\sum_{j=1}^{3} \bar{N}\left(r, \frac{1}{f-a_{j}}\right) & \leq N\left(r, \frac{1}{P(f)-f}\right) \\
& \leq T(r, f)+(n-1) N(r, f)+S_{q}(r, f), \quad r \geq R
\end{aligned}
$$

from above, it follows that

$$
\begin{aligned}
(2-\varepsilon) T(r, f) & \leq \bar{N}(r, f)+\sum_{j=1}^{3} \bar{N}\left(r, \frac{1}{f-a_{j}}\right) \\
& \leq(2-2 \varepsilon) T(r, f)+S_{q}(r, f),
\end{aligned}
$$

which is impossible. This contradiction yields $f \equiv P(f)$.

\section{Acknowledgment}

The first author was supported in part by 2012 Zhejiang Educational and Scientific Projects (SCG295).

\section{References}

[1] W. K. Hayman, Meromorphic Functions, Clarendon Press, Oxford, UK, 1964.

[2] C.-C. Yang and H.-X. Yi, Uniqueness Theory of Meromorphic Functions, Kluwer Academic, New York, NY, USA, 2003.

[3] I. Laine, Nevanlinna Theory and Complex Differential Equations, Walter de Gruyter, Berlin, Germany, 1993.

[4] W. K. Hayman, "Picard values of meromorphic functions and their derivatives," Annals of Mathematics, vol. 70, pp. 9-42, 1959.

[5] Y.-M. Chiang and S.-J. Feng, "On the Nevanlinna characteristic of $f(z+\eta)$ and difference equations in the complex plane," Ramanujan Journal, vol. 16, no. 1, pp. 105-129, 2008.

[6] J. Heittokangas, R. Korhonen, I. Laine, and J. Rieppo, "Uniqueness of meromorphic functions sharing values with their shifts," Complex Variables and Elliptic Equations, vol. 56, no. 1-4, pp. 81-92, 2011.

[7] K. Liu and I. Laine, "A note on value distribution of difference polynomials," Bulletin of the Australian Mathematical Society, vol. 81, no. 3, pp. 353-360, 2010. 
[8] Z.-X. Chen, "On value distribution of difference polynomials of meromorphic functions," Abstract and Applied Analysis, vol. 2011, Article ID 239853, 9 pages, 2011.

[9] D. C. Barnett, R. G. Halburd, R. J. Korhonen, and W. Morgan, "Nevanlinna theory for the $q$-difference operator and meromorphic solutions of q-difference equations," Proceedings of the Royal Society of Edinburgh A, vol. 137, no. 3, pp. 457-474, 2007.

[10] J. L. Zhang and R. Korhonen, "On the Nevanlinna characteristic of $f(q z)$ and its applications," Journal of Mathematical Analysis and Applications, vol. 369, no. 2, pp. 537-544, 2010.

[11] W. K. Hayman, "On the characteristic of functions meromorphic in the plane and of their integrals," Proceedings of the London Mathematical Society, vol. 14, pp. 93-128, 1965. 


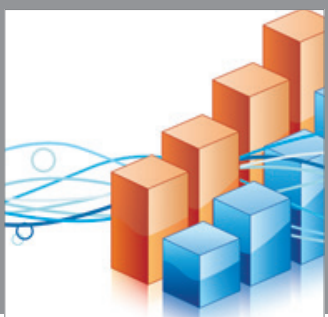

Advances in

Operations Research

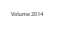

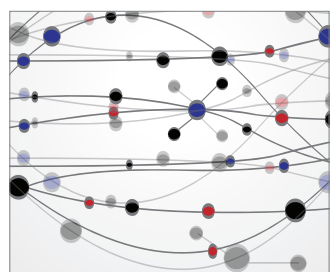

\section{The Scientific} World Journal
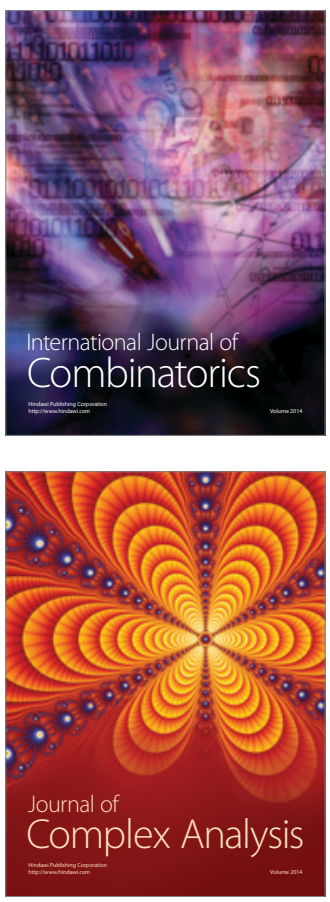

International Journal of

Mathematics and

Mathematical

Sciences
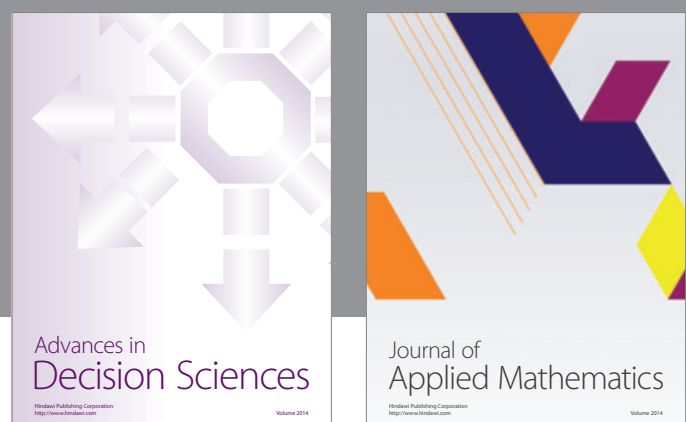

Journal of

Applied Mathematics
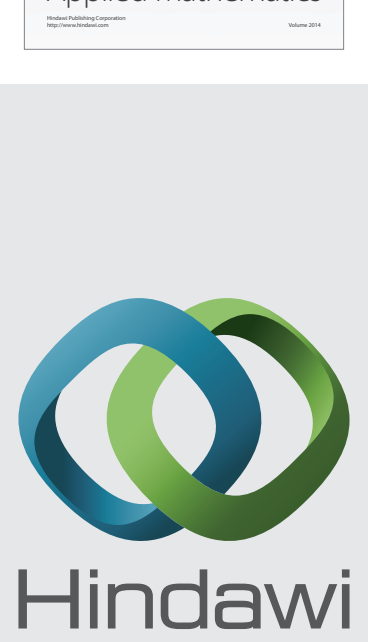

Submit your manuscripts at http://www.hindawi.com
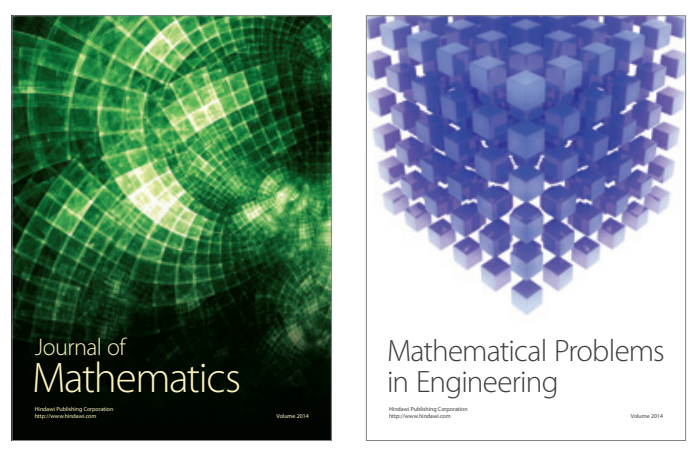

Mathematical Problems in Engineering
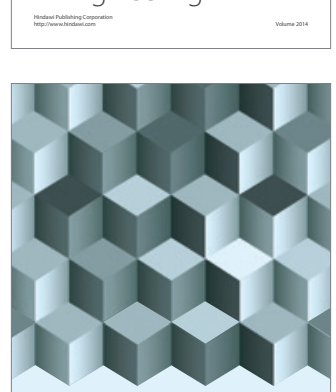

Journal of

Function Spaces
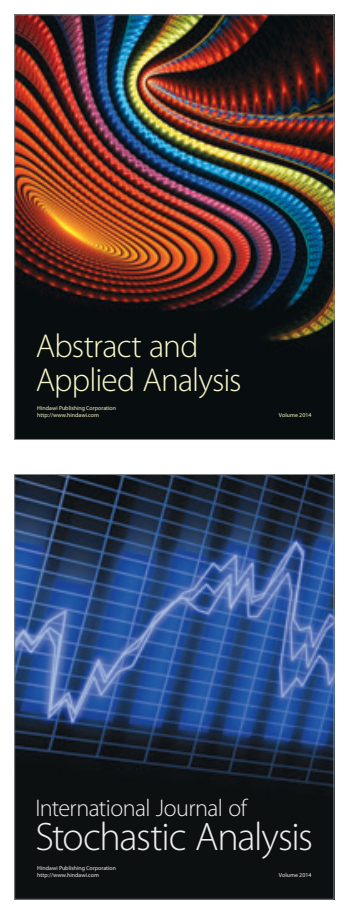

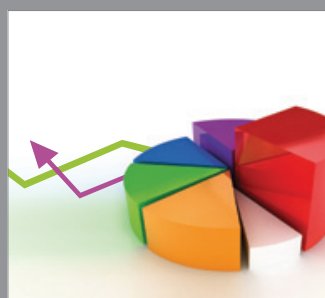

ournal of

Probability and Statistics

Promensencen
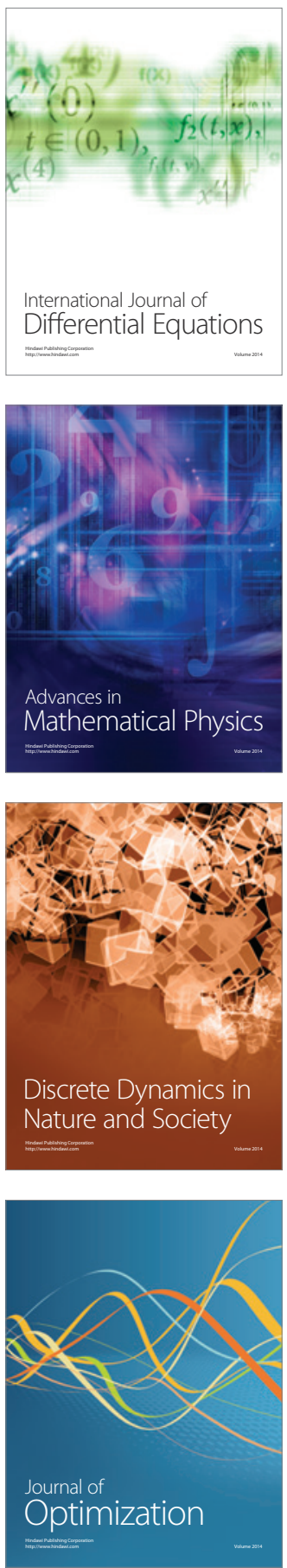